\title{
PáleS
}

You are invited to take part in a study

\section{The Project About Loneliness and Social \\ Networks (PALS)}

\section{A summary of the study}

- We are running a study to find out how our connections with other people and our community are important for our health and wellbeing.

- Half the people taking part in the study (Group 2) will be able to use a tool called GENIE now to map their support networks and match them with local activities. The other half will continue as usual but will be able to use GENIE later, after six months. The group you are in will be decided by chance.

- If you are over 18 and are interested in increasing your social contacts then please get in touch.

\section{If you take part in the PALS study}

The flowchart below shows you what the study involves and when things happen.

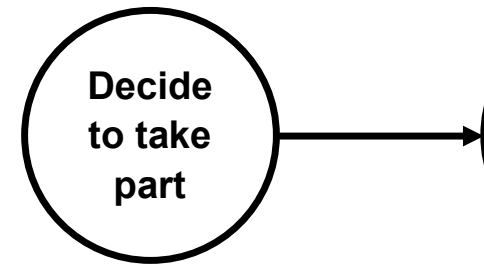

1.We will explain the study and ask for your consent. If you are in Group 2 we will help you to use GENIE.

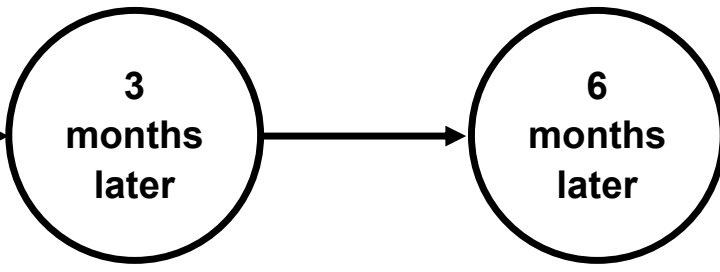

3.We will contact you to complete your last questionnaires. If you are you to complete your questionnaires. in Group 1, you will now be able to use GENIE.

\section{$\Rightarrow$ LIVERPOOL JOHN MOORES UNIVERSITY}

\section{Southampton}

PALS main study PIS

Version 4.0, 11Jun2018
UOS ERGO reference: 31638 IRAS reference: 232890 Page 1 of 8

\section{What is the study about?}

We want to find out how we can improve social contacts and activities of adults within local communities. We sometimes call this our social network.

We have created an online tool called GENIE to assess people's social network and link people with social activities and support in their local communities.

We need people to try out GENIE to see whether this helps improve their lives and wellbeing.

\section{Why have I been asked to take part?}

We are inviting anyone aged over $\mathbf{1 8}$ who is living in the community and is interested in increasing their social contacts to take part.

\section{What will I need to do if I want to take part?}

If you would like to take part please fill out the reply slip and send it to us in the FREEPOST envelope (no stamp is needed) to:

FAO PALS team

CLAHRC Wessex

Building 67, University of Southampton

University Road

Southampton

SO17 1BJ

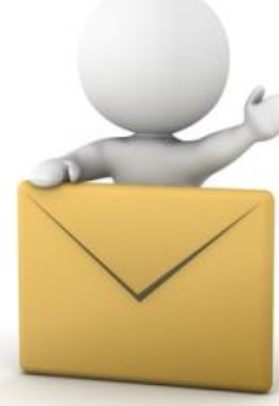




\section{What would I do in the study?}

After you return the reply slip to us, a researcher will call you to check you understand about the study and arrange a time to come and see you. This can be at your home or somewhere else convenient to you. At that meeting, the researcher will ask you to sign a consent form and you will fill out a questionnaire booklet. You will then find our which group you are in:

\section{Group 1}

Half the people in the study will be randomly chosen to be in Group 1, which means using GENIE in six-months time.

You will be sent another questionnaire booklet after three months and after six months. When you have completed all of these questionnaires the facilitator will get in touch with you to arrange a time for them to guide you through GENIE.

This group is very important to the study as it allows the researchers to compare people who are currently using GENIE and people who are not using GENIE.

\section{Group 2}

Half the people in the study will be randomly chosen to be in Group 2, which means using GENIE straight away.

Your facilitator will contact you to arrange a time to meet with you and guide you through GENIE. He/she will come back and see you after three months for a second visit.

You will be sent another questionnaire booklet after three months and after six months.

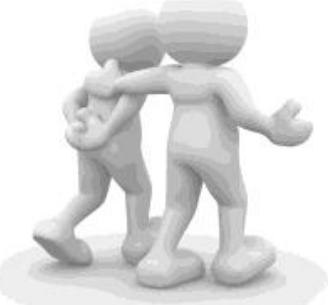

UoS ERGO reference: 31638 IRAS reference: 232890 Page 3 of 8

\section{What happens during GENIE?}

GENIE is an online tool that helps connect people to support and services around them with the aim of improving health and wellbeing.

You will think about all of the people and places that are important to you, which can include friends, neighbours and activities. GENIE will help you to create a visual map of these relationships.

You will answer some questions about your interests, support, health and wellness needs, and enjoyable activities. Then GENIE will show you a range of local community activities and support that fit in with your personal interests.

Using GENIE will take about 30 to 40 minutes.

Two weeks after using GENIE you will receive a phone call from the facilitator to see how things are going and if you are interested in any additional activities.

\section{$5 \quad$ What else can I do to help?}

You may also be asked to take part in an interview with a researcher so we can find out what you thought of GENIE and the social activities and support that it suggested for you.

You do not have to take part in this. If you choose to take part you will be given another information sheet to read and consent form to sign.

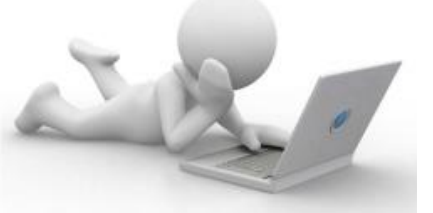


What are the possible pros and cons of taking part?

Taking part in the study could give you some good ideas of ways to increase your social connections and could have a positive impact on your health and wellbeing. You will also help researchers to better understand whether GENIE is a useful tool for people wanting to increase their social network. You will get a $\mathbf{f 1 0}$ gift voucher as a thank you for your help.

The main disadvantage of taking part is that it will take up some of your time. It will take about 30 minutes to fill in the questions (this is done at the beginning of the study, after three months, and after six months) and 30-40 minutes to go through GENIE.

You are unlikely to feel distressed during the study, but if you do then you can stop taking part at any time. If you continue to feel distressed after the study, the Samaritans offer confidential phone support around the clock (phone number: 116 123).

\section{More information about taking part}

Will my details be kept private? Anything you say to the researcher will be kept private. Confidentiality will only be broken if you tell the researcher something that causes them to worry that you or other people are at risk of harm. If this happens then the researcher will let the relevant authorities know (e.g. GP). Your answers to the questionnaires will be given a number so that your answers and other people's answers cannot be identified.

Can I change my mind? You can change your mind or stop at any time. You do not have to tell us why. If you stop it is up to you if we keep the information we have collected from you. You can choose to remove your data until it has been analysed.

How will my data be stored and who can see it?

All data collected during this study, including your contact details, will be entered onto a database. This is hosted by FormsVision and Interconnect BV, an ISO 9001 and 27001 certified provider. The actual data is held on a pair of servers in Holland, at a place called 's Hertogenbosch (generally known as Den Bosch). Each server is a mirror copy of the other, and the whole operation is EC and FDA compliant. A full description of the FormsVision Hosting Environment is available. Please ask if you would like to see a copy.

The information collected in this study will be looked at by researchers at the University of Southampton, your GENIE facilitator and may be looked at by regulatory authorities.

Only the trial team will have access to the database and they will only be able to access data that is relevant to their role. For example, the facilitator will be able to see your contact details so they can visit you, the administrator will be able to see your address so they can send you questionnaires, and the researchers will be able to see your questionnaires answers.

Paper copies of data, such as your reply slip, consent form and returned questionnaires will be stored in a locked filing cabinet in a locked office at the University of Southampton. The University of Southampton's policy is that all research data, along with signed consent forms, will be saved for 10 years and then destroyed.

We my contact you in the future if you say that you are happy for researchers to contact you about future studies on the consent form.

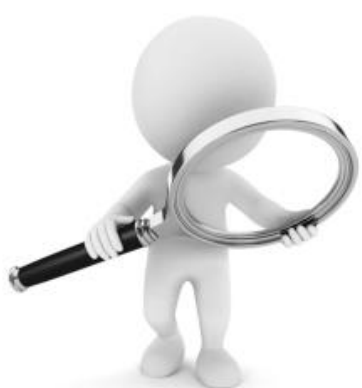

UOS ERGO reference: 31638 IRAS reference: 232890 Page 6 of 8 
What will happen to the results of the study? The results of the study will be written up and published. The results will also be discussed at conferences and other reports such as press releases. These will never include the names of people who have taken part, only code names or numbers. You can have a results summary if you wish. Who has reviewed the study? The study has been approved by the University of Southampton ethics committee and an NHS Research Ethics Committee (ethics reference numbers: 31638 and 232890).

Are we GDPR Compliant? Yes. The University of Southampton is the sponsor for this study based in the United Kingdom. We will be using information from you in order to undertake this study and will act as the data controller for this study. This means that we are responsible for looking after your information and using it properly. The University of Southampton will keep identifiable information about you for 10 years after the study has finished.

Your rights to access, change or move your information are limited, as we need to manage your information in specific ways in order for the research to be reliable and accurate. If you withdraw from the study, we will keep the information about you that we have already obtained. To safeguard your rights, we will use the minimum personally-identifiable information possible.

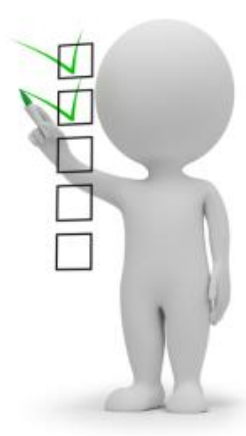

The University of Southampton will use your name, and contact details to contact you about the research study, and make sure that relevant information about the study is recorded for your care, and to oversee the quality of the study. Individuals from the University of Southampton and regulatory organisations may look at your research records to check the accuracy of the research study.

$\begin{array}{lr}\text { PALS main study PIS } & \text { UoS ERGO reference: } 31638 \\ \text { Version 4.0, 11Jun2018 } & \text { IRAS reference: } 232890 \\ & \text { Page } 7 \text { of } 8\end{array}$

The only people in the University of Southampton who will have access to information that identifies you will be people who need to contact you to or audit the data collection process. The people who analyse the information will not be able to identify you and will not be able to find out your name or contact details.

\section{Who is paying for and running the study?}

The study is being funded by the National Institute for Health Research, the main funder of research in the NHS. The study is being run by the University of Southampton.

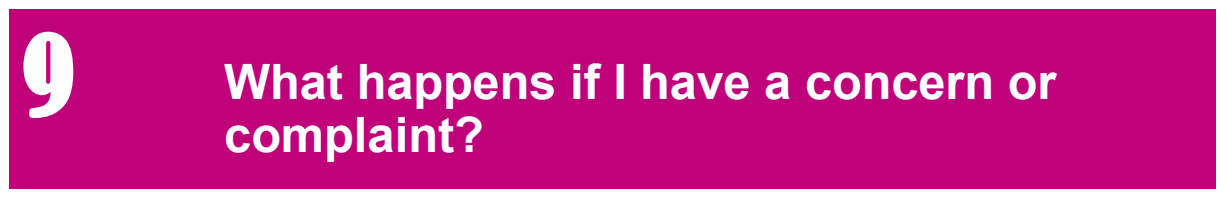

If you have a concern or complaint about the study, please contact the Research Integrity and Governance Manager (023 8059 5058, rgoinfo@soton.ac.uk).The University of Southampton has insurance in place to cover its legal liabilities in respect of this study.

Contact details
Phone: 02380598264
Email: include@soton.ac.uk

PALS main study PIS Version 4.0, 11Jun2018 